\title{
INPUT-TO-STATE STABILITY WITH RESPECT TO MEASUREMENT DISTURBANCES FOR ONE-DIMENSIONAL SYSTEMS *
}

\author{
Nicolas Chung Siong FaH ${ }^{1}$
}

\begin{abstract}
We consider one-dimensional affine control systems. We show that if such a system is stabilizable by means of a continuous, time-invariant feedback, then it can be made input-to-state stable with respect to measurement disturbances, using a continuous, periodic time-varying feedback. We provide counter-examples showing that the result does not generally hold if we want the feedback to be time-invariant or if the control system is not supposed affine.
\end{abstract}

Résumé. Nous étudions des systèmes de contrôle affines de dimension un. Nous montrons que si un tel système est stabilisable à l'aide d'un feedback continu indépendant du temps, alors il peut aussi être rendu "input-to-state stable" par rapport aux erreurs de mesure à l'aide d'un feedback continu dépendant périodiquement du temps. Nous donnons en outre des contre-exemples montrant que ce résultat est en général faux si on ne considère que des feedbacks indépendants du temps, ou si le système étudié n'est pas affine.

AMS Subject Classification. AMS: 93D25.

Received June 26, 1998. Revised February 15, 1999.

\section{INTRODUCTION}

When we study, in practice, the response of a feedback system to a control, we need to take into account exogenous disturbances that can be added to the system or the control. Stabilizability does provide natural robustness (see Sect. 2) on compact sets, but this robustness may decrease to zero as the size of the set grows to infinity. Input-to-state stability (ISS) was introduced by Sontag in [1] in order to deal with far from equilibrium robustness. Control disturbances, i.e. errors added to the control signal before it reaches the plant, have been widely studied, and we know from [1] that if an affine control system can be made globally asymptotically stable (GAS) with a time-invariant feedback, then it can also be made ISS with a (generally different) time-invariant feedback.

The same kind of study has been led for errors added to the measurement input before it reaches the controller, called measurement disturbances. A counter-example has shown (Freeman [2]) that there is generally no similar result in dimensions higher than 2. Nevertheless, a similar result does hold for those systems that can be put in strict feedback form (see [3]). The problem remains open for other systems.

Keywords and phrases: Input-to-state stability, stabilization, measurement errors.

* This work was partially supported by the CNRS GDR "Automatique".

1 École Normale Supérieure, 45 rue d'Ulm, 75005 Paris, France; e-mail: chung@clipper.ens.fr 
One-dimensional strict feedback form systems can be written as

$$
\dot{x}=f(x)+u g(x)
$$

where $x, u \in \mathbb{R}, f$ and $g$ are real continuous functions and moreover

$$
\forall y \in \mathbb{R}, g(y) \neq 0
$$

Freeman and Kokotovic prove that if such a system can be made GAS with a time-invariant continuous feedback, then it can be made ISS for measurement disturbances with a time-invariant continuous feedback.

In Section 2, we show that we can loosen restriction (1) to allow $g$ to have zeros, whose set we must nevertheless suppose bounded.

In Section 3, we give a counter-example showing that restriction (1) cannot be completely lifted, even we suppose $g$ of class $C^{\infty}$.

We show in Sections 4, 5 and 6 how to lift the restriction (1) if we allow the use of periodic time-varying feedbacks. Precisely, we prove the following theorem

Theorem 1. Consider the system

$$
\dot{x}=f(x)+u g(x)
$$

where $x \in \mathbb{R}$ is the state, $u \in \mathbb{R}$ the control, $f: \mathbb{R} \rightarrow \mathbb{R}$ and $g: \mathbb{R} \rightarrow \mathbb{R}$ are continuous.

Suppose there exists some continuous time-invariant feedback making the closed-loop plant GAS.

Then, for any period $T>0$, there exists a continuous T-periodic time-varying feedback making the closed-loop plant ISS with respect to measurement disturbances.

Moreover, if the set of $g$ 's zeros is bounded, the feedback can be taken time-invariant.

The interest of time-varying feedbacks compared to stationary feedbacks was first stressed when Sontag and Sussmann proved in [4] that one dimensional state nonlinear control systems which are controllable can made GAS by means of time-varying feedback laws. This result was extended to a nonholomic cart by Samson in [5] and to many systems in higher dimension by Coron in [6] and [7]. It was extended to output feedback laws by Coron in [8].

In [9], Freeman applies periodic-time varying feedbacks to affine systems for a slightly different problem. He studies ISS for measurement disturbances with systems that are only partially observable. More precisely, the sign of $g$ in equation (2) is still supposed constant, but unknown.

Finally, in Section 7, we provide a further counter-example showing that our result does not hold in general for non-affine control systems.

\section{THE CASE WHERE THE ZEROS OF G ARE BOUNDED}

A function $\gamma: \mathbb{R}^{+} \rightarrow \mathbb{R}$ is of class $\mathcal{K}$ if it is continuous, strictly increasing, and if $\gamma(0)=0$. The function $\gamma$ is of class $\mathcal{K}_{\infty}$ if moreover it is not bounded.

A function $\beta: \mathbb{R}^{+} \times \mathbb{R}^{+} \rightarrow \mathbb{R}^{+}$of class $\mathcal{K} \mathcal{L}$ if for each fixed $t$, the mapping $\beta(., t)$ is of class $\mathcal{K}$ and for each fixed $s$ the mapping $\beta(s,$.$) is decreasing to zero on t$ as $t \rightarrow+\infty$.

When $d \in L^{\infty}\left(\mathbb{R}^{+}\right)$, we note $\|d\|_{\infty}:=\operatorname{Sup}\{d(t) ; t \geq 0\}$.

ISS was first defined by Sontag in [1]. We adapt his definition to our problem of ISS of affine systems for measurement disturbances. From now on, ISS will mean input-to-state stability for measurement disturbances.

Definition 1. A time-invariant feedback $u \in C^{0}(\mathbb{R} ; \mathbb{R})$, such that $u(0)=0$, makes the system (2) ISS if and only if there exists a function $\gamma$ of class $\mathcal{K}$ and a function $\beta$ of class $\mathcal{K} \mathcal{L}$ such that any continuous solution of the closed-loop plant

$$
\dot{x}=f(x)+u(x+d) g(x)
$$


where $d: \mathbb{R}^{+} \rightarrow \mathbb{R}$ is a measurable essentially bounded function of $t$, exists for all $t \geq 0$, and satisfies

$$
|x(t)|<\beta(|x(0)|, t)+\gamma\left(\|d\|_{\infty}\right) .
$$

We also need the following characterization of ISS (Sontag and Wang [12]):

Proposition 1. A time-invariant feedback $u \in C^{0}(\mathbb{R}, \mathbb{R})$, such that $u(0)=0$, makes the system (2) ISS if and only if there exists a function $\gamma_{0}$ of class $\mathcal{K}$ and a function $\gamma_{1}$ of class $\mathcal{K}$ such that any solution of the closed-loop plant

$$
\dot{x}=f(x)+u(x+d) g(x),
$$

where $d: \mathbb{R}^{+} \rightarrow \mathbb{R}$, is a measurable essentially bounded function of $t$, exists for all $t \geq 0$, is bounded, and satisfies

$$
\|x\|_{\infty} \leq \gamma_{0}(|x(0)|)+\gamma_{1}\left(\|d\|_{\infty}\right)
$$

and

$$
\overline{\lim }_{t \rightarrow \infty}|x(t)| \leq \gamma_{1}\left(\overline{\lim }_{t \rightarrow \infty}|d(t)|\right) .
$$

We also use the following lemma due to Sontag [1]:

Lemma 1. Assume there exists a function $V: \mathbb{R} \rightarrow \mathbb{R}$, functions $\alpha_{1}$, $\alpha_{2}$ of class $\mathcal{K}_{\infty}$ such that

$$
\forall y \in \mathbb{R}, \alpha_{1}(|y|) \leq V(y) \leq \alpha_{2}(|y|),
$$

and $\gamma$ of class $\mathcal{K}$ such that, for any real number $\delta$ and $y$,

$$
|y|>\gamma(|\delta|) \Longrightarrow \frac{\partial V}{\partial y}(y)[f(y)+u(y+\delta) g(y)]<0 .
$$

Then the closed-loop system is ISS for measurement disturbances.

In the one-dimensional case, we can always take $V(y)=\alpha_{1}(y)=\alpha_{2}(y)=y^{2} / 2$.

Remark 1. We can assume with no loss of generality that $\gamma$ is greater than identity. This implies that we can separate the study for $x>0$ and for $x<0$. Proofs for $x<0$ can be deduced from those for $x>0$ by considering the system $\dot{x}=\tilde{f}(x)+u \tilde{g}(x)$, where $\tilde{f}(x)=-f(-x)$ and $\tilde{g}(x)=-g(-x)$.

We study system (2), and we suppose in this section that the set

$$
\{y \geq 0 ; g(y)=0\}
$$

is bounded. Let $a$ be $g$ 's largest positive zero if $g$ has a zero on $(0,+\infty)$, and let $a:=1$ otherwise.

Without any loss of generality, we can assume

$$
\forall y>a, g(y)>0 .
$$

We also assume that the system can be made GAS by means of a time-invariant, continuous feedback $w: \mathbb{R} \rightarrow \mathbb{R}$ such that $w(0)=0$.

Note $V$ the standard Lyapunov function $V(y):=\frac{y^{2}}{2}$. GAS is equivalent to $\dot{V}<0$ along the trajectories of $\dot{x}=f(x)+w(x) g(x)$. In particular,

$$
\forall y>0, f(y)+w(y) g(y)<0 .
$$

It is known that stabilizability provides us with some natural robustness to measurement disturbances. For example, we have in [10] or [11], 
Theorem 2. If $w$ is a continuous globally asymptotically stabilizing feedback, there exists a non-negative function $r_{m} \in C^{\infty}\left(\mathbb{R}^{+} \backslash\{0\} ; \mathbb{R}^{+} \backslash\{0\}\right) \cap C^{0}\left(\mathbb{R}^{+}\right)$such that, for any continuous $d: \mathbb{R} \rightarrow \mathbb{R}$ satisfying

zero is uniformly GAS for the system

$$
\forall y \in \mathbb{R},|d(y)| \leq r_{m}(y)
$$

$$
\dot{x}=f(x)+w(x+d(x)) g(x)
$$

Unfortunately, this is not enough to grant input-to-state stability, as $r_{m}(y)$ is allowed to tend to zero when $y$ grows to infinity. This stresses the fact that ISS is a problem at infinity. Indeed, any stabilizing feedback $w$ gives us some robustness for small initial conditions. Therefore, our main task consists in the construction of some feedback $v$ that provides us with Lemma 1 conditions for large $x$. We then show how to "glue together" $v$ and $w$ to get the ISS property.

Define $v: \mathbb{R}^{+} \rightarrow \mathbb{R}$ a continuous function such that $v(0):=0$ and satisfying the following property:

$$
\forall y>3 a, v(y) \leq \operatorname{Min}\left\{w(z) ; z \in\left[\frac{y}{2}, 2 y\right]\right\}
$$

Let $k \in C^{\infty}\left(\mathbb{R}^{+} ;[0,1]\right)$ be an increasing function such that

$$
\begin{aligned}
& \forall y \leq 9 a, k(y):=0 \\
& \forall y \geq 10 a, k(y):=1
\end{aligned}
$$

Then the feedback

$$
u:=(1-k) w+k v
$$

makes the closed-loop system ISS.

Indeed, the closed-loop system can be written as

$$
\dot{x}=(1-k(x))(f(x)+w(x) g(x))+k(x)(f(x)+v(x) g(x)) .
$$

Properties (6) to (11) show that $\forall x>0, x \dot{x}<0$, so that $u$ is indeed a stabilizing feedback. As the inequality (7) is strict, and all the functions continuous, we can find a function $r_{m} \in C^{0}\left(\mathbb{R}^{+} ; \mathbb{R}^{+}\right)$such

$$
\forall y>0, r_{m}(y)>0
$$

and

$$
\forall y>0, \forall|\delta| \leq r_{m}(y), y(f(y)+w(y+\delta) g(y))<0
$$

We choose $r_{m}$ strictly increasing on the interval $[0,20 a]$ and $r_{m}(0)=0$.

Finally, let $r$ be a continuous, non-negative, strictly increasing function such that

$$
\begin{aligned}
& \forall y \leq 20 a, r(y): \leq r_{m}(y) \\
& \forall y \geq 0, r(y): \leq y / 2 .
\end{aligned}
$$

and moreover $r(y) \rightarrow+\infty$ as $y \rightarrow+\infty$.

By (8),

$$
\forall y>6 a, \forall|\delta|<y / 2, v(y+\delta) \leq w(y)
$$


so that, with (6),

$$
\forall y \geq 6 a, \forall|\delta| \leq y / 2, y(f(y)+v(y+\delta) g(y)) \leq y(f(y)+w(y) g(y))<0
$$

By (10),

$$
\forall y \geq 20 a, \forall|\delta| \leq y / 2, k(y+\delta)=1
$$

With (12) and (13), we get

$$
\forall y \geq 0, \forall|\delta| \leq r(y),(1-k(y+\delta))(f(y)+w(y+\delta) g(y)) \leq 0 .
$$

Then, combining (12) and (15), and using the properties (9) and (10) of $k$, and the properties (13) and (14) of $r$, we get

$$
\forall y>0, \forall|\delta|<r(y), y(f(y)+u(y+\delta) g(y))<0
$$

Then $\gamma=r^{-1}$ is a class $\mathcal{K}$ function, and replacing $r$ by $\gamma^{-1}$ in (17) gives the property required in Lemma 1 for ISS.

\section{A COUNTER-EXAMPLE IN THE GENERAL CASE}

We consider equation (2), but we now allow $g$ to have a non-bounded set of zeros. We show in this section that we can pick $f$ and $g$ of class $C^{\infty}$ such that the system is stabilizable, but that no continuous time-invariant feedback can make the closed-loop system ISS. Our counter-example is based on the following idea: near a point $x$ where $f(x)=0$, if a stabilizing feedback's sign varies too quickly, we can always manage to construct a disturbance that will "choose" the wrong sign for $u$, and thus the wrong sign for $\dot{x}$, destroying the system's stability.

Take

$$
g(y)=\cos \left(y^{2}\right)
$$

and consider the points $x_{n}=\sqrt{n \pi}$. We have

$$
g\left(x_{n}\right)=(-1)^{n}
$$

Choose $f$ of class $C^{\infty}$ satisfying

$$
\begin{aligned}
& \forall n \in \mathbb{N}, f\left(x_{n}\right)=0, \\
& \forall y \geq 0, f(y) \leq 0, \\
& \forall y \geq 0, g(y)=0 \Rightarrow f(y)<0,
\end{aligned}
$$

e.g. $f(y)=-y \sin ^{2}\left(y^{2}\right)$. We check with $(20)$ and (21) that taking $u(y)=-y g(y)$ makes the closed-loop system globally asymptotically stable.

Let $w$ be a continuous time-invariant stabilizing feedback. The closed-loop system satisfies $\forall x \in \mathbb{R} \backslash\{0\}, \dot{x} x$ $<0$. Therefore, if $y \neq 0$ and $f(y)=0$, we must have $y w(y) g(y)<0$. In particular, with (18) and (19), we get $w\left(x_{n}\right) w\left(x_{n+1}\right)<0$ for all $n \in \mathbb{N}$. But for any arbitrary constant $\delta$, and a big enough $p \in \mathbb{N}$, we have $x_{p+1}-x_{p} \leq \delta$.

This implies that if we take $d_{p}(t)=x_{p+1}-x_{p}$ as the measurement disturbance, the closed-loop system $\dot{x}=f(x)+u\left(x+d_{p}\right) g(x)$ satisfies

$$
f\left(x_{p}\right)+w\left(x_{p}+d_{p}\right) g\left(x_{p}\right)>0
$$


Consequently, any trajectory of the closed-loop system such that $x(0) \geq x_{p}$ satisfies

$$
\forall t \geq 0, x(t) \geq x_{p}
$$

This construction is true for any large enough $p$, and $x_{p} \rightarrow+\infty$ when $p \rightarrow+\infty$. We have thus found disturbances $d_{p}$ such that

$$
\varlimsup_{t \rightarrow \infty}\left|d_{p}(t)\right| \rightarrow 0 \text { when } p \rightarrow+\infty
$$

and corresponding trajectories $y_{p}$ such that

$$
\varlimsup_{t \rightarrow \infty}\left|y_{p}(t)\right| \rightarrow+\infty \text { when } p \rightarrow+\infty \text {. }
$$

Therefore, there cannot be any function $\gamma_{1}$ of class $\mathcal{K}$ such that (4) is satisfied, and our system cannot be made ISS using a continuous time-invariant feedback.

\section{Construction of AN ISS time-VArying FEedBack}

In a time-varying framework, definition 1 is no longer adequate. A definition of ISS for time-varying systems was given by Lin in [13].

Definition 2. A feedback $u \in C^{0}(\mathbb{R} \times \mathbb{R}, \mathbb{R})$, such that for all $t \in \mathbb{R}, u(0, t)=0$, makes the system (2) ISS if and only if there exists a function $\gamma$ of class $\mathcal{K}$ and a function $\beta$ of class $\mathcal{K} \mathcal{L}$ such that any solution of the closed-loop plant

$$
\dot{x}=f(x)+u(x+d, t) g(x),
$$

where $d$ is a measurable essentially bounded disturbance, and with the initial condition

$$
x\left(t_{0}\right)=x_{0}
$$

exists for all $t \geq t_{0}$, and satisfies

$$
|x(t)|<\beta\left(\left|x_{0}\right|, t-t_{0}\right)+\gamma\left(\left\|d^{t_{0}}\right\|_{\infty}\right),
$$

where $d^{t_{0}}$ is the function defined by $d^{t_{0}}(t):=0$ if $t<t_{0}$, and $d^{t_{0}}(t):=d(t)$ for $t \geq t_{0}$.

We study the control system (2), that we suppose GAS by means of a continuous time-invariant feedback. Let

$$
Z:=\left\{y \in \mathbb{R}^{+} ; g(y)=0\right\} \text {. }
$$

In Sections 4 and 5, we assume that for some $\alpha>0, Z \cap[\alpha,+\infty)$ is a discrete and infinite set, and can thus be described as a strictly increasing, unbounded sequence $\left(a_{n}\right)_{n \in \mathbb{N}}$. The other cases shall be treated in Section 6 . As we said in Remark 1, we treat explicitly only the case $x \geq 0$. We can assume with no loss of generality that

$$
\forall y \in\left(a_{0}, a_{1}\right), g(y)>0 .
$$

As the system is stabilizable, and as $f$ is continuous, $y f(y)<0$ in neighbourhoods of positive real numbers where $\mathrm{g}$ is zero. In particular,

$$
\forall n>0, \forall u \in \mathbb{R}, f\left(a_{n}\right)+u g\left(a_{n}\right)=f\left(a_{n}\right)<0 .
$$

This gives us one of the system's key properties: whatever feedback we may apply, for any solution of the control system (2)

$$
\left(x\left(t_{0}\right) \leq a_{n}\right) \Rightarrow\left(\forall t \geq t_{0}, x(t) \leq a_{n}\right) .
$$


The strategy of the proof consists in constructing a periodic time-varying feedback, of arbitrary chosen period $T>0$, so that the closed-loop system satisfies the following property:

$$
\forall n>0,0 \leq x(0) \leq a_{n+1} \Rightarrow 0 \leq x(T) \leq a_{n}
$$

All trajectories are forced in finite time into the interval $\left(0, a_{1}\right)$. The trajectories near zero are then stabilized like in Section 2.

The main difficulty we face when we allow $g$ 's zeros to be unbounded is the following. In Section 2 , equations (8) and (11) show us how to modify any stabilizing feedback $w$ to enforce robustness: for large $x$, replace $w$ by $\operatorname{Min}\left\{w(y) ; y \in\left[\frac{x}{2}, 2 x\right]\right\}$. Of course, this construction relied on the fact that $g$ is positive on $(a,+\infty)$. If we had supposed $g$ negative on $(a,+\infty)$, we should have taken $\operatorname{Max}\left\{w(y) ; y \in\left[\frac{x}{2}, 2 x\right]\right\}$ instead. But in the new situation, where $g$ does not have a fixed sign near infinity, neither of these modifications works. However, if we allow the feedbacks to be periodic time-varying, we will be able treat separately in time the regions where $g$ is non-negative, and those where $g$ is non-positive. Equation (25) will once again be decisive in assuring us that while we treat trajectories situated in the regions where $g$ is positive, no great harm can be done to the trajectories passing through regions where $g$ is negative.

Let $T>0$ be our feedback's period. Let $w$ be some continuous time-invariant feedback stabilizing system (2) such that $w(0)=0$. As in the previous section, $w$ will provide robustness near zero. Like in (12), we can find a continuous function $r_{m}: \mathbb{R}^{+} \rightarrow \mathbb{R}$ such that $r_{m}(y)>0$ for $y>0$, and

$$
\forall y>0, \forall \delta \in \mathbb{R},|\delta| \leq r_{m}(y) \Rightarrow f(y)+w(y+\delta) g(y)<0
$$

We can suppose that $r_{m}$ is strictly increasing on $\left[0, a_{1}\right]$ and that

$$
\forall y \in \mathbb{R}^{+}, r_{m}(y) \leq \frac{y}{2}
$$

Recall from (24) that $f$ is continuous and negative in the neighbourhood of the points $a_{n}$. We can thus define a function $B:\left(a_{n}\right)_{n \in \mathbb{N}} \rightarrow \mathbb{R}$ so that, for $n>0, B\left(a_{n}\right)<a_{n}$ is a point such that

$$
\left(\dot{x}=f(x), x(0) \leq a_{n}\right) \Rightarrow\left(x(T / 6) \leq B\left(a_{n}\right)\right)
$$

and a function $C:\left(a_{n}\right)_{n \in \mathbb{N}} \rightarrow \mathbb{R}$ so that, for $n \geq 0, C\left(a_{n}\right)>a_{n}$ is a point such that:

$$
\left(\dot{x}=f(x), x(0) \leq C\left(a_{n}\right)\right) \Rightarrow\left(x(T / 6) \leq a_{n}\right) .
$$

We choose these points so that $\forall n \geq 0, C\left(a_{n}\right)<B\left(a_{n+1}\right)$, and so that

$$
\forall n \geq 1, \forall y \in\left[B\left(a_{n}\right), C\left(a_{n}\right)\right], f(y)<0 .
$$

Let $B\left(a_{0}\right):=0$.

Let $\tilde{v} \in C^{0}\left(\mathbb{R}^{+}, \mathbb{R}^{-}\right)$be such that

$$
\forall n \geq 0, \forall y \in\left[C\left(a_{n}\right), B\left(a_{n+1}\right)\right], \tilde{v}(y) \leq-2 \frac{\left(B\left(a_{n+1}\right)-C\left(a_{n}\right)\right) \frac{6}{T}+\operatorname{Max}(f(y), 0)}{|g(y)|}
$$

Then let $v$ be defined as:

$$
\forall y \geq 0, v(y):=\operatorname{Min}\{\tilde{v}(z) ; z \in[y / 2,2 y]\}
$$


The function $v$ is also continuous and non-positive, and

$$
\forall y>0, \forall \delta \in[-y / 2, y / 2], v(y+\delta) \leq \tilde{v}(y) .
$$

The function $\sin (2 \pi t / T) v$ provides robustness far from zero.

As in Section 2, we must define a function $k$ that will "glue" $w$ and $v$ together: equation (23) gives us

$$
\forall y \in\left[a_{0}, a_{1}\right], \forall t \in[0, T / 2], \sin (2 \pi t / T) \tilde{v}(y) g(y) \leq 0 .
$$

As $f\left(a_{1}\right)<0$, we can therefore define $p \in \mathbb{R}$ a point such that $a_{0}<p<a_{1}$, and $t_{1} \in \mathbb{R}$ a time such that $0<t_{1}<T / 2$, and

$$
\left(\dot{x}=\frac{1}{2}(f(x)+\sin (2 \pi t / T) \tilde{v}(x) g(x)), x(0) \leq a_{1}\right) \Rightarrow\left(x\left(t_{1}\right) \leq p\right) .
$$

Properties (23) and (32) imply that, if $d$ is a measurable essentially bounded function and $x$ a continuous function such that

$$
\begin{gathered}
\dot{x}(t)=\frac{1}{2}(f(x(t))+\sin (2 \pi t / T) v(x(t)+d(t)) g(x(t))), \\
x(0) \leq a_{1}, \\
|d(t)| \leq|x(t)| / 2 \text { for } t \text { a.e. in } \mathbb{R}^{+},
\end{gathered}
$$

then

$$
x\left(t_{1}\right) \leq p .
$$

Likewise, inequality (26) allows us to define a point $q \in \mathbb{R}$ and a time $t_{2} \in \mathbb{R}$ such that $a_{0}<q<p$ and $0<t_{1}<t_{2}$, and, if $d$ is a measurable essentially bounded function and $x$ a continuous function such that

$$
\begin{gathered}
\dot{x}(t)=\frac{1}{2}(f(x(t))+w(x(t)+d(t)) g(x(t))), \\
x\left(t_{1}\right) \leq p, \\
|d(t)| \leq r_{m}(x(t)) \text { for } t \text { a.e. in } \mathbb{R}^{+},
\end{gathered}
$$

then

$$
x\left(t_{2}\right) \leq q .
$$

As $f\left(a_{1}\right)<0$, we can choose $p$ and $q$ such that

$$
\forall q \leq y \leq a_{1}, f(y)<0 .
$$

Let $k$ be an increasing function in $C^{\infty}(\mathbb{R},[0,1])$ satisfying

$$
\begin{cases}\forall y \leq q+\left(\frac{p-q}{3}\right), & k(y):=0, \\ \forall q+\left(\frac{p-q}{3}\right) \leq y \leq p-\left(\frac{p-q}{3}\right), & 0 \leq k(y) \leq \frac{1}{2} \\ \forall p-\left(\frac{p-q}{3}\right) \leq y \leq p+\left(\frac{a_{1}-p}{3}\right), & k(y):=\frac{1}{2}, \\ \forall p+\left(\frac{a_{1}-p}{3}\right) \leq y \leq a_{1}-\left(\frac{a_{1}-p}{3}\right), & \frac{1}{2} \leq k(y) \leq 1 \\ \forall a_{1}-\left(\frac{a_{1}-p}{3}\right) \leq y, & k(y):=1 .\end{cases}
$$


Let $r$ be a function of class $\mathcal{K}_{\infty}$ such that

$$
\begin{gathered}
\forall y \geq 0, r(y) \leq y / 2, \\
\forall y \leq a_{1}, r(y) \leq r_{m}(y),
\end{gathered}
$$

and, moreover,

$$
\begin{aligned}
& \forall y \geq a_{1}, r(y) \leq y-a_{1}+\frac{a_{1}-p}{3}, \\
& \forall y \in\left[p, a_{1}\right], r(y) \leq y-p+\frac{p-q}{3}, \\
& \forall y \in[q, p], r(y) \leq p-y+\frac{a_{1}-p}{3}, \\
& \forall y \in\left[a_{0}, q\right], r(y) \leq q-y+\frac{p-q}{3} .
\end{aligned}
$$

Then the feedback

$$
u(x, t):=\sin (2 \pi t / T) v(x) k(x)+w(x)(1-k(x))
$$

makes the closed-loop system ISS.

\section{Proof of Theorem 1 in the Discrete CASE}

Lemma 2. For all $n>0$, if $x: \mathbb{R}^{+} \rightarrow \mathbb{R}$ and $d: \mathbb{R}^{+} \rightarrow \mathbb{R}$ satisfy

$$
\begin{gathered}
\dot{x}=f(x)+u(x+d, t) g(x) \\
0 \leq x(0) \leq a_{n+1} \\
a_{n} \leq x(t) \leq a_{n+1} \Rightarrow|d(t)| \leq r(x(t)) \text { for } t \text { a.e in } \mathbb{R}^{+}
\end{gathered}
$$

then we have $x(T) \leq a_{n}$.

Proof. The result follows immediately from $(25)$ when $x(0) \leq a_{n}$. Now suppose $a_{n}<x(0) \leq a_{n+1}$. First consider the case when

$$
\forall y \in\left(a_{n}, a_{n+1}\right), g(y)>0 .
$$

The definition (43) of $k$ and (50) of $u$ imply that

$$
\forall y \geq a_{1}-\left(\frac{a_{1}-p}{3}\right), u(y, t)=\sin (2 \pi t / T) v(y) .
$$

Then (32), (46) and (55) give

$$
\begin{aligned}
& \left(y>a_{1},|\delta| \leq r(y), t \in[0, T / 2]\right) \Rightarrow u(y+\delta, t) \leq 0, \\
& \left(y>a_{1},|\delta| \leq r(y), t \in[T / 2, T]\right) \Rightarrow u(y+\delta, t) \geq 0 .
\end{aligned}
$$

Recall that $a_{n}<C\left(a_{n}\right)<B\left(a_{n+1}\right)<a_{n+1}$ (see (28) and (27)).

First, we show that $x(T / 6) \leq B\left(a_{n+1}\right)$. Indeed, either $x(0) \leq B\left(a_{n+1}\right)$ and, since (29), (54), and (56) give us

$$
\forall t \in[0, T / 2], \forall|\delta| \leq r\left(B\left(a_{n+1}\right)\right), f\left(B\left(a_{n+1}\right)\right)+u\left(B\left(a_{n+1}\right)+\delta, t\right) g\left(B\left(a_{n+1}\right)\right)<0,
$$


we have $x(T / 6) \leq B\left(a_{n+1}\right)$.

Or $B\left(a_{n+1}\right)<x(0)<a_{n+1}$. Then (54) and (56) implies

$$
\forall t \in[0, T / 6], \forall y \in\left[B\left(a_{n+1}\right), a_{n+1}\right], \forall|\delta| \leq r(y), f(y)+u(y+\delta, t) g(y) \leq f(y),
$$

which, with (27), implies that $\exists \tau \in[0, T / 6)$ such that $x(\tau)=B\left(a_{n+1}\right)$. Finally, (58) shows that $x(T / 6)$ $\leq B\left(a_{n+1}\right)$.

Then, $x(T / 3) \leq C\left(a_{n}\right)$. Indeed, either $x(T / 6) \leq C\left(a_{n}\right)$, and since (29), (54), and (56) give us

$$
\forall t \in[0, T / 2], \forall|\delta| \leq r\left(C\left(a_{n}\right)\right), f\left(C\left(a_{n}\right)\right)+u\left(C\left(a_{n}\right)+\delta, t\right) g\left(C\left(a_{n}\right)\right)<0,
$$

we have $x(T / 3) \leq C\left(a_{n}\right)$.

Or $C\left(a_{n}\right)<x(T / 6)<B\left(a_{n+1}\right)$. Equation (55) shows that when $y>a_{1}$ and $t \in[T / 6, T / 3]$,

$$
u(y, t) \leq \frac{v(y)}{2} .
$$

We deduce from (32) that

$$
\forall y>a_{1}, \forall|\delta| \leq r(y), \forall t \in[T / 6, T / 3], u(y+\delta, t) \leq \frac{\tilde{v}(y)}{2},
$$

so that the definition (30) of $\tilde{v}$ and (54) give us

$$
\begin{aligned}
\forall y \in\left[C\left(a_{n}\right), B\left(a_{n+1}\right)\right], \forall|\delta| & \leq r(y), \forall t \in[T / 6, T / 3], f(y)+u(y+\delta, t) g(y) \\
& \leq f(y)-\left(B\left(a_{n+1}\right)-C\left(a_{n}\right)\right) \frac{6}{T}-\operatorname{Max}(f(y), 0) \\
& \leq-\left(B\left(a_{n+1}\right)-C\left(a_{n}\right)\right) \frac{6}{T} .
\end{aligned}
$$

If we integrate this last inequality along a trajectory $x$, we find that

$$
\exists \tau \in(T / 6, T / 3), x(\tau)=C\left(a_{n}\right),
$$

and (60) finally shows that $x(T / 3) \leq C\left(a_{n}\right)$.

Then, $x(T / 2) \leq a_{n}$. Indeed, either $x(T / 3) \leq a_{n}$, and (25) implies that $x(T / 2) \leq a_{n}$.

Or $a_{n}<x(T / 3)<C\left(a_{n}\right)$, then (29), (54), and (56) give us

$$
\forall t \in[0, T / 2], \forall y \in\left[a_{n}, C\left(a_{n}\right)\right], \forall|\delta| \leq r(y), f(y)+u(y+\delta, t) g(y) \leq f(y),
$$

which, with (28), implies the existence of $\tau$ in $(T / 3, T / 2)$ such that $x(\tau)=a_{n}$. Equation (25) then gives us $x(T / 2) \leq a_{n}$.

Finally, the same equation (25) shows that $x(T) \leq a_{n}$.

If $g$ is negative on $\left(a_{n}, a_{n+1}\right)$, we still have $x(T / 2) \leq a_{n+1}$, and, the same argument used above for $g>0$ and $t \in[0, T / 2]$ shows that $x(T) \leq a_{n}$, which ends the proof of Lemma 2 .

We now show a similar Lemma for $n=0$ :

Lemma 3. If $x: \mathbb{R}^{+} \rightarrow \mathbb{R}$ and $d: \mathbb{R}^{+} \rightarrow \mathbb{R}$ satisfy

$$
\begin{gathered}
\dot{x}=f(x)+u(x+d, t) g(x) \\
0 \leq x(0) \leq a_{1}, \\
q \leq x(t) \leq a_{1} \Rightarrow|d(t)| \leq r(x(t)) \text { for } t \text { a.e in } \mathbb{R}^{+}
\end{gathered}
$$

then we have $x(T) \leq q$. 
Proof. From (42), (23), and (32) we get

$$
\forall y \in\left[q, a_{1}\right], \forall|\delta| \leq r(y), \forall t \in[0, T / 2], f(y)+\sin (2 \pi t / T) v(y+\delta) g(y)<0 .
$$

Properties (26) and (44) imply that

$$
\forall y \in\left[q, a_{1}\right], \forall|\delta| \leq r(y), f(y)+w(y+\delta) g(y)<0 .
$$

We first prove that $x\left(t_{1}\right) \leq p$ (the time $t_{1}$ was defined in (33)). Indeed, either $x(0) \leq p$, and the definition (50) of $u$, along with (67) and (68) show that

$$
\forall t \in[0, T / 2], \forall|\delta| \leq r(p), f(p)+u(p+\delta, t) g(p)<0 .
$$

Therefore, $x\left(t_{1}\right) \leq p$.

Or $p<x(0) \leq a_{1}$. Then, for all $y \in\left[p, a_{1}\right]$ and all $|\delta| \leq r(y)$, the property (47) of $r$ shows that $y+\delta \geq p-\frac{p-q}{3}$, so that (43) gives us $k(y+\delta) \geq \frac{1}{2}$. This and equation (68) imply that

$$
\forall t \in[0, T / 2], f(y)+u(y+\delta, t) g(y) \leq \frac{1}{2}(f(y)+\sin (2 \pi t / T) v(y+\delta) g(y)) .
$$

If we integrate this inequality along the trajectory $x$, properties (37) and (69) show that $x\left(t_{1}\right) \leq p$.

We next show that $x\left(t_{2}\right) \leq q$. Indeed, either $x\left(t_{1}\right) \leq q$, and the definition (50) of $u$, along with (67) and (68) show that

$$
\forall t \in[0, T / 2], \forall|\delta| \leq r(q), f(q)+u(q+\delta, t) g(q)<0
$$

Therefore, $x\left(t_{1}\right) \leq q$.

Or $q<x\left(t_{1}\right) \leq p$. Then, for all $y \in[q, p]$ and all $|\delta| \leq r(y)$, the property (48) of $r$ shows that $y+\delta \leq p+\frac{a_{1}-p}{3}$, so that (43) gives us $k(y+\delta) \leq \frac{1}{2}$. This and equation (67) imply that

$$
\forall t \in[0, T / 2], f(y)+u(y+\delta, t) g(y) \leq \frac{1}{2}(f(y)+w(y+\delta) g(y)) .
$$

If we integrate this inequality along the trajectory $x$, properties (41) and (71) show that $x\left(t_{2}\right) \leq q$.

Finally, (71) shows that $x(T / 2) \leq q$, which ends the proof of Lemma 3.

We now define the functions $\beta$ and $\gamma$ needed in Definition 2 for the ISS property. Properties (45) and (26) show that

$$
\forall 0<y \leq q, \forall|\delta| \leq r(y), f(y)+w(y+\delta) g(y)<0 .
$$

Like in Lemma 1, this implies that if $x$ is a solution of the equation $\dot{x}=f(x)+w(x+d) g(x)$, where $d$ is a measurable essentially bounded disturbance such that $\|d\|_{\infty} \leq r(q)$, and if $0 \leq x(0) \leq q$, then there exists a function $\beta_{0}$ of class $\mathcal{K} \mathcal{L}$ and a function $\gamma_{0}$ of class $\mathcal{K}_{\infty}$ such that

$$
\forall t \geq 0,|x(t)| \leq \beta_{0}(x(0), t)+\gamma_{0}\left(\|d\|_{\infty}\right) .
$$

We also suppose that

$$
\forall x \in[0, q], \forall t \leq 0, \beta_{0}(x, t)<a_{1} .
$$

Definition 3. Let $\beta$ be a function of class $\mathcal{K} \mathcal{L}$ such that: 
- If $a_{n}<y \leq a_{n+1}$ and $n>0$,

$$
\left\{\begin{array}{l}
\forall t \in[0,2 T], \beta\left(y, t-t_{0}\right) \leq a_{n+1} \\
\forall 1 \leq k \leq n, \forall t \in[(k+1) T,(k+2) T], \beta\left(y, t-t_{0}\right) \leq a_{n-k+1} \\
\forall t \geq(n+2) T, \beta\left(y, t-t_{0}\right) \geq \beta_{0}(q, t-(n+2) T) .
\end{array}\right.
$$

- If $q<y \leq a_{1}$,

$$
\begin{cases}\forall t \in[0,2 T], & \beta\left(y, t-t_{0}\right) \leq a_{1} \\ \forall t \geq 2 T, & \beta\left(y, t-t_{0}\right) \geq \beta_{0}(q, t) .\end{cases}
$$

- If $0 \leq y \leq q$, then $\forall t \geq 0, \beta\left(y, t-t_{0}\right) \geq \beta_{0}\left(y, t-t_{0}\right)$.

Note that (74) is needed for this definition to make sense on $\left[q, a_{1}\right]$.

Definition 4. Let $\gamma$ be a continuous function of class $\mathcal{K}_{\infty}$ such that

$$
\begin{cases}\forall n>0, \forall \delta \in\left[r\left(a_{n}\right), r\left(a_{n+1}\right)\right], & \gamma(\delta) \geq a_{n+1} \\ \forall \delta \in\left[r(q), r\left(a_{1}\right)\right), & \gamma(\delta) \geq a_{1} \\ \forall \delta \in[0, r(q)), & \gamma(\delta) \geq \gamma_{0}(\delta) .\end{cases}
$$

These two functions satisfy the requirements of Definition 2 of ISS.

Indeed, let $d: \mathbb{R}^{+} \rightarrow \mathbb{R}$ be a measurable, essentially bounded measurement disturbance and let $x: \mathbb{R}^{+} \rightarrow \mathbb{R}$ be a trajectory of the closed-loop system

$$
\begin{aligned}
\dot{x}(t) & =f(x(t))+u(x(t)+d(t), t) g(x(t)) \\
x\left(t_{0}\right) & =x_{0}>0
\end{aligned}
$$

where $t_{0} \in[0, T)$, and $x_{0} \geq 0$. Define $n \in \mathbb{N}$ so that $a_{n}<x_{0} \leq a_{n+1}$. First suppose that $n>0$.

Case 1. If $\|d\|_{\infty} \geq r\left(a_{n}\right)$, then $\gamma\left(\|d\|_{\infty}\right) \geq a_{n+1}$. But (25) shows that for all times $t \geq t_{0},|x(t)| \leq a_{n+1}$, so that $|x(t)| \leq \gamma\left(\|d\|_{\infty}\right) \leq \beta\left(x_{0}, t-t_{0}\right)+\gamma\left(\|d\|_{\infty}\right)$.

Case 2. If there exists an integer $1 \leq p \leq n$ such that $\|d\|_{\infty} \in\left[r\left(a_{p-1}\right), r\left(a_{p}\right)\right)$ and if $\|d\|_{\infty}>r(q)$, then (25) shows that $x(T) \leq a_{n+1}$ and Lemma 2 shows that for all integer $j \in[0, n+1-p]$ and all times $t \in$ $[(j+1) T,(j+2) T), x(t) \leq a_{n+1-j}$. We can check with the definition of $\beta$ that for all times $t \in\left[t_{0}, T+(n+2-p) T\right)$, $|x(t)| \leq \beta\left(x_{0}, t-t_{0}\right) \leq \beta\left(x_{0}, t-t_{0}\right)+\gamma\left(\|d\|_{\infty}\right)$.

Then (25) shows that for all times $t \geq T+(n+2-p) T, x(t) \leq a_{p}$ and we can check with the definition of $\gamma$ that $x(t) \leq \gamma\left(\|d\|_{\infty}\right) \leq \beta\left(x_{0}, t-t_{0}\right)+\gamma\left(\|d\|_{\infty}\right)$.

Case 3. If $\|d\|_{\infty} \leq r(q)$, like in the previous paragraph, Lemma 2 and the definition of $\beta$ shows that for all times $t \in\left[t_{0},(n+2) T\right),|x(t)| \leq \beta\left(x_{0}, t-t_{0}\right) \leq \beta\left(x_{0}, t-t_{0}\right)+\gamma\left(\|d\|_{\infty}\right)$. Moreover, $x((n+1) T) \leq a_{1}$, and Lemma 3 shows that $x((n+2) T) \leq q$. The bound on $d$ and the definition of $u$ then shows that for times $t \geq(n+2) T$, the trajectory $x$ obeys the equation $\dot{x}=f(x)+w(x+d) g(x)$.

Finally, (73) shows that for all times $t \geq(n+2) T,|x(t)| \leq \beta_{0}(x(n+2) T, t-(n+2) T)+\gamma_{0}\left(\|d\|_{\infty}\right) \leq \beta_{0}(q, t-$ $(n+2) T)+\gamma_{0}\left(\|d\|_{\infty}\right) \leq \beta\left(x_{0}, t-t_{0}\right)+\gamma\left(\|d\|_{\infty}\right)$. Therefore, the trajectories all satisfy property (22).

In the cases where $x_{0} \in\left(q, a_{1}\right]$ or $x_{0} \in[0, q]$, the same arguments as above show that the trajectories also satisfy (22). Therefore, $u$ does make the closed-loop system ISS.

\section{The General CASE}

At this point, we have only completed our proof for $Z$ bounded or discrete near infinity. Let us treat straight away a couple of other cases: 
1. If $g \equiv 0$, there is nothing to do. The system being GAS is naturally ISS.

2. If there exists some $b>0$ such that $g \equiv 0$ on $[b,+\infty)$, it turns out that we do not need a time-varying feedback. As a matter of fact, the situation is very similar to that studied in Section 2. Using

$$
\forall y \in[b,+\infty), \forall u \in \mathbb{R}, f(y)+u g(y)=f(y)<0,
$$

the proof goes along the same lines if we just replace the function $v$ defined in (8) by the zero function. From now on, we suppose that $Z$ is not discrete and that for all $A>0$, there are some real numbers $a>A$ and $b>A$ such that $g(a) \neq 0$ and $g(b)=0$.

In this last case, the strategy will be the following: for some $\underline{a}$ to be defined, we select from $Z \cap[\underline{a},+\infty)$ a discrete, strictly increasing, unbounded subset $\left(z_{n}\right)_{n \in \mathbb{N}}$ in an appropriate way. Then if we define $u$ as if $\left(z_{n}\right)_{n \in \mathbb{N}}$ were the set of $g$ 's zero, like in the previous section (with a few modifications), the trajectories of the closed-loop system (with measurement disturbances) satisfy the property

$$
\forall n>0, \exists j \in \mathbb{N}, x(0) \leq z_{n+1} \Rightarrow x(j T) \leq z_{n},
$$

allowing us to conclude in the same way.

\subsection{Selecting a "significant" subset of zeros}

Choose $a$ so that we can define $I:=(\underline{a}, \bar{a})$ the biggest subinterval of $\mathbb{R}^{+}$such that $a \in I$ and $\forall y \in I, g(y) \neq 0$, and such that $\underline{a}>0$ and $g(\underline{a})=g(\bar{a})=0$. Let

$$
Z^{\prime}=Z \cap[\underline{a},+\infty) .
$$

We shall use the following conventions:

$$
\begin{aligned}
& \operatorname{Sup}\{y ; y \in \emptyset\}=-\infty \\
& \operatorname{Inf}\{y ; y \in \emptyset\}=+\infty .
\end{aligned}
$$

Definition 5. For each $z \in Z$, define a "following zero" $F(z) \in Z$ :

Case 1. If Inf $\{g(y)=0 ; y>z\}>z$, we say that $z$ is (an) isolated (zero) on its right. Then let

$$
F(z):=\operatorname{Min}\{g(y)=0 ; y>z\}
$$

Case 2. If $\operatorname{Inf}\{g(y)=0 ; y>z\}=z$, and $\operatorname{Inf}\{g(y) \neq 0 ; y>z\}>z$, we say that $z$ has an interval of zeros on its right. Then let

$$
F(z):=\operatorname{Inf}\{g(y) \neq 0 ; y>z\}
$$

i.e. $F(z)$ is the largest real number such that

$$
\forall y \in[z, F(z)], g(y)=0,
$$

so that on intervals of time where $x(t) \in[z, F(z)]$, the trajectory satisfies the equation

$$
\dot{x}=f(x) .
$$

Moreover, on that compact interval, $f$ is negative, so that there exists some integer $j>0$ such that

$$
x(0) \leq F(z) \Rightarrow x((j-1) T) \leq z .
$$


Case 3. If $\operatorname{Inf}\{g(y)=0 ; y>z\}=z$, and $\operatorname{Inf}\{g(y) \neq 0 ; y>z\}=z$, we say that $z$ is an accumulation point (of zeros) on its right. As $f(z)<0$ and $f$ is continuous, there exists some point $\zeta>z$, such that

$$
(\dot{x}=f(x), x(0)=\zeta) \Rightarrow x(T / 12)<z .
$$

As we assumed that $\operatorname{Inf}\{g(y)=0 ; y>z\}=\operatorname{Inf}\{g(y) \neq 0 ; y>z\}=z$, we can suppose that $g(\zeta) \neq 0$, and we can define

$$
F(z):=\operatorname{Sup}\{g(y)=0 ; y<\zeta\}
$$

Moreover, this $F(z)$ satisfies

$$
(\dot{x}=f(x), x(0)=F(z)) \Rightarrow x(T / 12)<z .
$$

Likewise,

Definition 6. Define a "previous zero" $P(z) \in Z$ for all $z \in Z$ :

Case 1. If $\operatorname{Sup}\{g(y)=0 ; y<z\}<z$, we say that $z$ is (an) isolated (zero) on its left. Then let

$$
\begin{gathered}
P(z):=\operatorname{Max}\{g(y)=0 ; y<z\} \text { if this real number exists } \\
P(z):=0 \text { otherwise. }
\end{gathered}
$$

Case 2. If $\operatorname{Sup}\{g(y)=0 ; y<z\}=z$, and $\operatorname{Sup}\{g(y) \neq 0 ; y<z\}<z$, we say that $z$ has an interval of zeros on its left. Then let

$$
P(z):=\operatorname{Sup}\{g(y) \neq 0 ; y<z\},
$$

so that on intervals of time where $x(t) \in[z, F(z)]$, the trajectory satisfies the equation

$$
\dot{x}=f(x) .
$$

Moreover, on that compact interval, $f$ is negative, so that there exists some integer $j>0$ such that

$$
x(0) \leq z \Rightarrow x((j-1) T) \leq P(z) .
$$

Case 3. If $\operatorname{Sup}\{g(y)=0 ; y<z\}=z$, and $\operatorname{Sup}\{g(y) \neq 0 ; y<z\}=z$, we say that $z$ is an accumulation point (of zeros) on its left. As $f(z)<0$ and $f$ is continuous, there exists some point $\zeta<z$, such that

$$
(\dot{x}=f(x), x(0)=z) \Rightarrow x(T / 12)<\zeta .
$$

As we assumed that $\operatorname{Sup}\{g(y)=0 ; y<z\}=\operatorname{Sup}\{g(y) \neq 0 ; y<z\}=z$, we can suppose that $g(\zeta) \neq 0$, and we can define

$$
P(z):=\operatorname{Inf}\{g(y)=0 ; y>\zeta\}
$$

Moreover, this $P(z)$ satisfies

$$
(\dot{x}=f(x), x(0)=z) \Rightarrow x(T / 12)<P(z) .
$$

Finally, let $P(0):=-1$. 
Clearly, $\bigcup_{z \in Z^{\prime}}(P(z), F(z)) \supset[\underline{a},+\infty)$. For each $n \in \mathbb{N}$, we can extract from the compact interval $[\underline{a}+n, \underline{a}+$ $n+1] \cap Z^{\prime}$ a finite family $Z_{n}^{\prime}$ such that $\bigcup_{z \in Z_{n}^{\prime}}(P(z), F(z)) \supset[\underline{a}+n, \underline{a}+n+1]$. Therefore, we can extract a countable, discrete subset of $Z^{\prime}$, that we note $\left(z_{n}\right)_{n \in \mathbb{N}}$, such that $z_{n+1}>z_{n}$ for all $n \in \mathbb{N}$ and

$$
\bigcup_{n \in \mathbb{N}}\left(P\left(z_{n}\right), F\left(z_{n}\right)\right) \supset[\underline{a},+\infty) .
$$

This subset can be chosen such that $z_{0}=\underline{a}, z_{1}=\bar{a}$. It is also very important to choose this subset as minimal in the sense that there is no smaller subset of $Z^{\prime}$ satisfying the conditions stated above.

Finally, we prove two technical lemmas.

Lemma 4. There is no $n \in \mathbb{N}$ such that there exists an non-empty open interval I such that $z_{n} \in I$ and $I \subset Z^{\prime}$.

Consequently, if $z_{n}$ has an interval of zeros on its right, it cannot have an interval of zeros on its left, and $\left[z_{n}, F\left(z_{n}\right)\right]$ is a maximal subinterval of $Z^{\prime}$. Likewise, if $z_{n}$ has an interval of zeros on its left, it cannot have an interval of zeros on its right, and $\left[P\left(z_{n}\right), z_{n}\right]$ is a maximal subinterval of $Z^{\prime}$.

Proof. If there is such an $I$, we can suppose it maximal in the sense that there is no greater open interval included in $Z^{\prime}$ and containing $z_{n}$. Then the definitions of $P\left(z_{n}\right)$ and $F\left(z_{n}\right)$ show that $I=\left(P\left(z_{n}\right), F\left(z_{n}\right)\right)$. Let $j<n$ be an integer. If $z_{j} \in I$, then $\left(P\left(z_{j}\right), F\left(z_{j}\right)\right)=I$. The minimal character of $\left(z_{n}\right)_{n \in \mathbb{N}}$ therefore implies that $z_{j} \leq P\left(z_{n}\right)$. Likewise, if $j>n, z_{j} \geq F\left(z_{n}\right)$.

As we have (89), either there is some $j<n$ such that $F\left(z_{j}\right)>P\left(z_{n}\right)$, or there is some $j>n$ such that $P\left(z_{j}\right)<F\left(z_{n}\right)$. Suppose we are in the first case (the proof for the second case goes along the same lines). Then we also have

$$
F\left(z_{j}\right)<F\left(z_{n}\right)
$$

otherwise $\left(P\left(z_{n}\right), F\left(z_{n}\right)\right) \subset\left(P\left(z_{j}\right), F\left(z_{j}\right)\right)$, which is impossible due to the minimal character of $\left(z_{n}\right)_{n \in \mathbb{N}}$. Thus, $F\left(z_{j}\right) \in I$.

But this situation is only possible if $z_{j}$ has a whole interval of zeros on its right. Indeed, if $z_{j}$ were isolated on its right, then (77) would imply that $F\left(z_{j}\right)$ is isolated on its left. That would contradict $F\left(z_{j}\right) \in I$. If $z_{j}$ were an accumulation point on its right, then (82) would define $F\left(z_{j}\right)$ as a Sup of points $y$ such that $g(y)=0$, and $y<\zeta$, where $g(\zeta) \neq 0$. As $g$ is zero in $I$, that would imply $F\left(z_{j}\right) \geq F\left(z_{n}\right)$, which would contradict $F\left(z_{j}\right) \in I$.

Then, according to (79), $\left[z_{j}, F\left(z_{j}\right)\right] \in Z^{\prime}$, and moreover $F\left(z_{j}\right)$ is the largest number such that this property is true. As $I=\left(P\left(z_{n}\right), F\left(z_{n}\right)\right), z_{j} \leq P\left(z_{n}\right)$, and $F\left(z_{j}\right)>P\left(z_{n}\right)$, we must have $F\left(z_{j}\right)=F\left(z_{n}\right)$, which contradicts (90), and finishes the proof of Lemma 4.

Lemma 5. We can choose the sequences $\left(z_{n}\right)_{n \in \mathbb{N}}, P\left(z_{n}\right)_{n \in \mathbb{N}}$, and $F\left(z_{n}\right)_{n \in \mathbb{N}}$ such that all three are strictly increasing.

Proof. The way we constructed $\left(z_{n}\right)_{n \in \mathbb{N}}$ does not automatically grant this property for the sequences or previous and next zeros. More precisely, using the definition of $P$, the minimal character of $\left(z_{n}\right)_{n \in \mathbb{N}}$ and Lemma 4 make it easy to check in each case, except when $z_{n}$ and $z_{n+1}$ are accumulation points on their left, that we do have $P\left(z_{n}\right)<P\left(z_{n+1}\right)$. In the same way, $F\left(z_{n}\right)<F\left(z_{n+1}\right)$, except when $z_{n}$ and $z_{n+1}$ are accumulation points on their right. Note that if $P\left(z_{n}\right) \geq P\left(z_{n+1}\right)$, then necessarily $F\left(z_{n}\right) \geq F\left(z_{n+1}\right)$ otherwise $\left(P\left(z_{n}\right), F\left(z_{n}\right)\right) \subset$ $\left(P\left(z_{n+1}\right), F\left(z_{n+1}\right)\right)$, which would contradict the minimal character of $\left(z_{n}\right)_{n \in \mathbb{N}}$. Of course, the symmetrical assertion is also true. Therefore, the only case where we do not have $P\left(z_{n}\right)<P\left(z_{n+1}\right)$ and $F\left(z_{n}\right)<F\left(z_{n+1}\right)$ is the case where $z_{n}$ and $z_{n+1}$ are accumulation points on both sides. 
But we can get rid of such cases the following way: if ever $P\left(z_{N}\right) \geq P\left(z_{N+1}\right)$ and $F\left(z_{N}\right) \geq F\left(z_{N+1}\right)$, we then see that

$$
[\underline{a},+\infty) \subset \bigcup_{0 \leq n \leq N-1}\left(P\left(z_{n}\right), F\left(z_{n}\right)\right) \cup\left(P\left(z_{N+1}\right), F\left(z_{N}\right)\right) \cup \bigcup_{n \geq N+2}\left(P\left(z_{n}\right), F\left(z_{n}\right)\right) .
$$

Moreover, as $z_{N}<z_{N+1},(82)$ shows that $F\left(z_{N}\right)$ also satisfies

$$
\dot{x}=f(x), x(0)=F\left(z_{N}\right) \Rightarrow x(T / 12)<z_{N+1} .
$$

Therefore, if you replace the sequence $\left(z_{n}\right)_{n \in \mathbb{N}}$ by the sequence $\left(z_{n}\right)_{n \in \mathbb{N} \backslash\{N\}}$, and $F\left(z_{N+1}\right)$ by $F\left(z_{N}\right)$, you obtain a new subsequence of $Z^{\prime}$ and new functions $F$ and $P$ that satisfy the same properties as the original ones. We can repeat this transformation in an ordered way (starting with $N=0$ ) each time we need to. Therefore, we can always suppose that $\left(z_{n}\right)_{n \in \mathbb{N}}$ is such that, for all integer $n$,

$$
\begin{aligned}
& P\left(z_{n}\right)<P\left(z_{n+1}\right), \\
& F\left(z_{n}\right)<F\left(z_{n+1}\right),
\end{aligned}
$$

which ends the proof of this lemma.

The minimality of $\left(z_{n}\right)_{n \in \mathbb{N}}$, along with properties (89), (91), and (92), then show that

$$
F\left(z_{n}\right)>P\left(z_{n+1}\right) .
$$

\subsection{Defining the feedback}

The definition goes quite along the same lines as Section 4. Define functions $w$ and $r_{m}$ like in (26). For all $z \in Z$, we have $f(z)<0$ and can therefore define some points $B(z)<z$ and $C(z)>z$ such that:

$$
(\dot{x}=f(x), x(0) \leq z) \Rightarrow x(T / 12)<B(z)
$$

and

$$
(\dot{x}=f(x), x(0) \leq C(z)) \Rightarrow x(T / 12)<z .
$$

Without any loss of generality, we can suppose that, for all $n \in \mathbb{N}$,

$$
z_{n}<C\left(z_{n}\right)<B\left(z_{n+1}\right)<z_{n+1} .
$$

Then let $\tilde{v}:[0,+\infty) \rightarrow(-\infty, 0]$ be a continuous, non-positive function such that, for all $n \geq 0$ :

1. If $\forall y \in\left(z_{n}, z_{n+1}\right), g(y) \neq 0$ (the case we dealt with in Sect. 5), then

$$
\forall n \geq 0, \forall y \in\left[C\left(z_{n}\right), B\left(z_{n+1}\right)\right], \tilde{v}(y) \leq-2 \frac{\left(B\left(z_{n+1}\right)-C\left(z_{n}\right)\right) \frac{6}{T}+\operatorname{Max}(f(y), 0)}{|g(y)|} .
$$

2. If $z_{n}$ is an accumulation point on its right, and if $z_{n+1}$ is isolated on its left, $\forall y \in\left(P\left(z_{n+1}\right), z_{n+1}\right), g(y) \neq 0$, so that, necessarily $P\left(z_{n+1}\right)>z_{n}$. Then let $\tilde{v}$ satisfy

$$
\forall n \geq 0, \forall y \in\left[C\left(P\left(z_{n+1}\right)\right), B\left(z_{n+1}\right)\right], \tilde{v}(y) \leq-2 \frac{\left(B\left(z_{n+1}\right)-C\left(P\left(z_{n+1}\right)\right)\right) \frac{6}{T}+\operatorname{Max}(f(y), 0)}{|g(y)|} .
$$


Define $v, p, q$, and $k$ like in section 4 (see respectively (31), (33), (41), and (43)), and let

$$
u(x, t):=m(t)(\sin (2 \pi t / T) v(x) k(x)+w(x)(1-k(x))),
$$

where $m$ is a $T$-periodic function of class $C^{\infty}$ such that

$$
\forall t \in \mathbb{R}, 0 \leq m(t) \leq 1,
$$

and

$$
\begin{cases}\forall t \in[0, T / 12], & m(t):=0, \\ \forall t \in[T / 6,5 T / 6], & m(t):=1, \\ \forall t \in[11 T / 12, T), & m(t):=0 .\end{cases}
$$

In particular, $\forall t \in[0, T / 12] \cup[11 T / 12, T]$, any trajectory $x$ of the closed-loop system satisfies

$$
\dot{x}=f(x) .
$$

Finally, define $r$ like in Section 4, satisfying properties (44) to (49).

\subsection{Proof of theorem 1}

Lemma 6. For all $n>0$, if $x: \mathbb{R}^{+} \rightarrow \mathbb{R}$ and $d: \mathbb{R}^{+} \rightarrow \mathbb{R}$ satisfy

$$
\begin{gathered}
\dot{x}=f(x)+u(x+d, t) g(x) \\
0 \leq x(0) \leq z_{n+1} \\
\forall t \geq 0, z_{n} \leq x(t) \leq z_{n+1} \Rightarrow|d(t)| \leq r(x(t)),
\end{gathered}
$$

then there exists some integer $j>0$ so that $x(j T) \leq z_{n}$.

Proof. The result follows immediately from (25) when $x(0) \leq z_{n}$. Now suppose $z_{n}<x(0) \leq z_{n+1}$.

The proof depends on the nature of $z_{n}$ and $z_{n+1}$ :

- If $z_{n}$ is isolated on its right, and $z_{n+1}$ is isolated on its left, then, according to (77) and (83), $\forall y \in$ $\left(z_{n}, F\left(z_{n}\right)\right), g(y) \neq 0$, and $\forall y \in\left(P\left(z_{n+1}\right), z_{n+1}\right), g(y) \neq 0$. With (93), we deduce that for all $y \in\left(z_{n}, z_{n+1}\right), g(y)$ $\neq 0$. In that case, the definition (96) of $\tilde{v}$ is the same than in Section 4 , and we prove in the same way as Lemma 2 that if $x(0) \leq z_{n+1}$, then $x(T) \leq z_{n}$.

- If $z_{n}$ is an accumulation point on its right, and $z_{n+1}$ is isolated on its left, then, with the definition (97) of $\tilde{v}$, we prove in the same way as in Lemma 2 that if $x(0) \leq z_{n+1}$, then

$$
x(T) \leq P\left(z_{n+1}\right) .
$$

Therefore, according to (93), $x(T) \leq F\left(z_{n}\right)$, which, with (82) and (100), shows that $x(13 T / 12) \leq z_{n}$. Finally, with (25), we can conclude that

$$
x(2 T) \leq z_{n} .
$$

- If $z_{n}$ has an interval of zeros on its right, and $z_{n+1}$ is isolated on its left. We show that this situation cannot occur. Indeed, (79) gives

but (83) implies that

$$
\forall y \in\left(z_{n}, F\left(z_{n}\right)\right), g(y)=0
$$

$$
\forall y \in\left(P\left(z_{n+1}\right), z_{n+1}\right), g(y) \neq 0 .
$$

As we supposed $z_{n+1}>z_{n}$, this implies that $P\left(z_{n+1}\right) \geq F\left(z_{n}\right)$, which contradicts (93). 
- If $z_{n}$ is isolated on its right and $z_{n+1}$ is an accumulation point on its left, we can deduce from (88) and (100) that

$$
x(T / 12) \leq P\left(z_{n+1}\right)
$$

But (77) shows that $\forall y \in\left(z_{n}, F\left(z_{n}\right)\right), g(y) \neq 0$. As $P\left(z_{n+1}\right) \in Z$, (93) shows that $P\left(z_{n+1}\right) \leq z_{n}$. Therefore, with (25), we get

$$
x(T) \leq z_{n}
$$

- If $z_{n}$ is an accumulation point on its right and $z_{n+1}$ is an accumulation on its left, then, according to (88), (100), and (93),

$$
x(T / 12) \leq P\left(z_{n+1}\right)<F\left(z_{n}\right) .
$$

As $F\left(z_{n}\right) \in Z$, we can deduce from $(25)$ that $x(11 T / 12) \leq F\left(z_{n}\right)$. Finally, we use (82) and (100) to show that

$$
x(T) \leq z_{n}
$$

- If $z_{n}$ has an interval of zeros on its right and $z_{n+1}$ is an accumulation point on its left, then, according to (88), (100), and (93), $x(T / 12) \leq P\left(z_{n+1}\right)<F\left(z_{n}\right)$. With (80), we can conclude that there is some integer $j>0$ such that

$$
x(j T) \leq z_{n}
$$

- If $z_{n}$ is isolated on its right and $z_{n+1}$ has an interval of zeros on its left: this situation cannot occur for the same reasons as its symmetrical situation.

- If $z_{n}$ is an accumulation point on its right and $z_{n+1}$ has an interval of zeros on its left (86) and (93) shows that there is some integer $j>0$ such that

$$
x((j-1) T) \leq P\left(z_{n+1}\right)<F\left(z_{n}\right) .
$$

Then (82) implies that $x((j-1) T+T / 12) \leq z_{n}$, so that

$$
x(j T) \leq z_{n}
$$

- If $z_{n}$ has an interval of zeros on its right and $z_{n+1}$ has an interval of zeros on its left, we see from (79), (85), and (93) that $\forall y \in\left[z_{n}, z_{n+1}\right], g(y)=0$, and the same argument as the one we used to prove (80) shows that there is some integer $j>0$ such that

$$
x(j T)<z_{n}
$$

which concludes the proof of Lemma 6.

Lemma 3 still holds in the general case with the same proof. We then define functions $\beta$ and $\gamma$ like in Section 4 and prove in the same way that they satisfy the requirements of the definition of ISS.

\section{A NON-LINEAR COUNTER-EXAMPLE}

When, in Section 3, we provided our counter-example, we stressed the role played by frequent oscillations in the sign of $g$. The proof relied on the fact that a "good" feedback for some point $x$ was not "good" anymore for a neighbour point, when $x$ is large enough. Using a similar idea, we now prove that a (non-affine) system GAS by means of a continuous time-invariant feedback cannot generally be made ISS for measurement disturbances, even if we allow periodic time-varying feedbacks.

What saves ISS in the affine case is the fact that an appropriate feedback can bring back $a_{n+1}$ to $a_{n}$ in an arbitrary (small) lapse of time. By taking a large $u$ of the right sign, one can get an arbitrary large $\dot{x}$ (except when $g$ is zero) of any sign. This property can of course no longer be granted for general nonlinear systems. We are therefore led to consider systems such that $f(x, u)$ has an finite upper or lower bound when $u \in \mathbb{R}$, a bound which vanishes to zero when $x \rightarrow \infty$. 
Theorem 3. The one-dimensional control plant

$$
\dot{x}=f(x, u)=\left(u-\sin \left(x^{2}\right)\right)^{2}-\frac{x}{3\left(x^{4}+3\right)},
$$

is GAS by means of a time-invariant continuous feedback, but cannot be made ISS for measurement disturbances by means of a periodic time-varying continuous feedback.

Proof. The feedback $w(y):=\sin \left(y^{2}\right)$ makes the closed-loop system GAS.

Consider a continuous, periodic time-varying feedback $u(x, t)$ of given period $T>0$ making the closed-loop system GAS, and let $\delta>0$ be the bound allowed for measurement disturbances, i.e. $\|d\|_{\infty} \leq \delta$.

Let

$$
b_{n}:=\sqrt{2 \pi n}
$$

for $n \in \mathbb{N}$. There exists an integer $N_{0}$ such that

$$
\forall n \geq N_{0}, b_{n+1}-b_{n}<\delta .
$$

Define

$$
\begin{aligned}
& A_{n}:=\left\{t \in[0,+\infty), \exists y \in\left[b_{n}, b_{n+1}\right], u(y, t) \geq 0\right\}, \\
& B_{n}:=\left\{t \in[0,+\infty), \exists y \in\left[b_{n}, b_{n+1}\right], u(y, t) \leq 0\right\},
\end{aligned}
$$

and $m_{A_{n}}:=\operatorname{meas}\left(A_{n} \cap[0, T]\right), m_{B_{n}}:=\operatorname{meas}\left(B_{n} \cap[0, T]\right)$.

Finally, introduce the points

$$
\begin{aligned}
x_{n} & :=\sqrt{\left(2 n+\frac{3}{2}\right) \pi}, \\
y_{n} & :=\sqrt{\left(2 n+\frac{1}{2}\right) \pi} .
\end{aligned}
$$

Let us now give some useful estimates. The function $x \mapsto \frac{x}{3\left(x^{4}+3\right)}$ increases from 0 to $\frac{1}{12}$ on the interval $[0,1]$, then decreases to 0 on $[1, \infty)$. Therefore, we have the global estimate

$$
\forall y \geq 0, \quad \forall u \in \mathbb{R}, \quad f(y, u) \geq-\frac{1}{12},
$$

and, with (105), for $n \geq 1, y \in\left[b_{n}, b_{n+1}\right]$, and $u \in \mathbb{R}$,

$$
f(y, u) \geq-\frac{y}{3\left(y^{4}+3\right)} \geq-\frac{b_{n}}{3\left(b_{n}{ }^{4}+3\right)}=-\frac{\sqrt{2 \pi n}}{3\left(4 \pi^{2} n^{2}+3\right)} .
$$

The following estimates hold when $n$ grows to infinity:

$$
\frac{\sqrt{2 \pi n} T}{3\left(4 \pi^{2} n^{2}+3\right)} \sim \frac{T}{3(2 \pi)^{3 / 2}} n^{-3 / 2}, x_{n}-\sqrt{\left(2 n+\frac{7}{6}\right) \pi} \sim \sqrt{\left(2 n+\frac{11}{6}\right) \pi}-x_{n} \sim \frac{\pi}{6 \sqrt{2 \pi}} n^{-1 / 2},
$$

and

$$
y_{n}-\sqrt{\left(2 n+\frac{1}{6}\right) \pi} \sim \sqrt{\left(2 n+\frac{5}{6}\right) \pi}-y_{n} \sim \frac{\pi}{6 \sqrt{2 \pi}} n^{-1 / 2}
$$


These estimates imply the existence of an integer $N_{1}$ such that, for all $n \geq N_{1}$ :

$$
\begin{aligned}
& \frac{\sqrt{2 \pi n} T}{3\left(4 \pi^{2} n^{2}+3\right)} \leq x_{n}-\sqrt{\left(2 n+\frac{7}{6}\right) \pi}, \\
& \frac{\sqrt{2 \pi n} T}{3\left(4 \pi^{2} n^{2}+3\right)} \leq \sqrt{\left(2 n+\frac{11}{6}\right) \pi}-x_{n},
\end{aligned}
$$

and

$$
\begin{aligned}
& \frac{\sqrt{2 \pi n} T}{3\left(4 \pi^{2} n^{2}+3\right)} \leq y_{n}-\sqrt{\left(2 n+\frac{1}{6}\right) \pi}, \\
& \frac{\sqrt{2 \pi n} T}{3\left(4 \pi^{2} n^{2}+3\right)} \leq \sqrt{\left(2 n+\frac{5}{6}\right) \pi}-y_{n} .
\end{aligned}
$$

Choose

$$
n \geq \operatorname{Max}\left(N_{0}, N_{1}\right) .
$$

It appears from (107) and (108) that $m_{A_{n}}+m_{B_{n}} \geq T$, so that one of the two sets has a measure greater than $T / 2$. Suppose for example that

$$
m_{A_{n}} \geq T / 2 \text {. }
$$

Let $\alpha: \mathbb{R}^{+} \rightarrow\left[b_{n}, b_{n+1}\right]$ be a measurable T-periodic function such that

$$
\forall t \in A_{n}, u(\alpha(t), t) \geq 0 .
$$

Then consider a continuous trajectory $s_{\tau}$ of the closed-loop system:

$$
\dot{x}=f(x, u(\alpha(t), t))
$$

with the initial condition $s_{\tau}(\tau)=x_{n}$.

Lemma 7. Then $s_{\tau}(T+\tau)>s_{\tau}(\tau)$.

Indeed, if we integrate (120) between $\tau$ and $T+\tau$, using estimates (112) and (113), and the definition (109) of $x_{n}$, we find that

$$
\forall t \in[\tau, T+\tau], s_{\tau}(t) \geq \sqrt{\left(2 n+\frac{7}{6}\right) \pi}
$$

There are two cases:

1. Either $\exists t_{0} \in(\tau, T+\tau]$ such that $s_{\tau}\left(t_{0}\right)=\sqrt{\left(2 n+\frac{11}{6}\right) \pi}$, then integrate (120) between $t_{0}$ and $T+\tau$, using estimates (112) and (114), as well as (109), to find that

$$
s_{\tau}(T+\tau)>s_{\tau}(\tau) .
$$


2. Or $\forall t \in[\tau, T+\tau], s_{\tau}(t) \leq \sqrt{\left(2 n+\frac{11}{6}\right) \pi}$.

But then, for all $y \in\left[\sqrt{\left(2 n+\frac{7}{6}\right) \pi}, \sqrt{\left(2 n+\frac{11}{6}\right) \pi}\right]$, we have $\sin y^{2} \leq-\frac{1}{2}$. If moreover $u \geq 0$, then

$$
f(y, u) \geq f(y, 0) \geq\left(\sin y^{2}\right)^{2}-\frac{y}{3\left(y^{4}+3\right)} \geq \frac{1}{4}-\operatorname{Max}\left\{\frac{z}{3\left(z^{4}+3\right)} ; z \geq 0\right\} \geq \frac{1}{4}-\frac{1}{12}=\frac{1}{6},
$$

which, with (111), gives us:

$$
s_{\tau}(T+\tau)-s_{\tau}(\tau)=\int_{\tau}^{\tau+T} \dot{s_{\tau}}=\int_{A_{n} \cap[\tau, \tau+T]} \dot{s_{\tau}}+\int_{[\tau, T+\tau] \backslash A_{n}} \dot{s_{\tau}} \geq m_{A_{n}} \frac{1}{6}-\left(T-m_{A_{n}}\right) \frac{1}{12} .
$$

As we supposed in (118) that $m_{A_{n}}$ is greater than $T / 2$, we finally get

$$
s_{\tau}(T+\tau)-s_{\tau}(\tau)>0
$$

The same lemma can be proved when $n$ is such that $m_{B_{n}}$ is greater than $T / 2$ : just consider the trajectories of the same closed-loop system, but with initial condition $s_{\tau}(\tau)=y_{n}$. The proof goes along the same lines, using estimates (115) and (116) instead of (113) and (114).

We now construct a measurable, essentially bounded disturbance $d$ and a trajectory $x$ of the closed-loop system

$$
\dot{x}=f(x, u(x+d, t))
$$

of initial condition $x(0)=x_{n}$ if $m_{A_{n}} \geq T / 2$ (resp. $x(0)=y_{n}$ if $m_{B_{n}} \geq T / 2$ ), such that the limsup of this trajectory is greater than $x_{n}$ (resp. $y_{n}$ ). We treat the case $m_{A_{n}} \geq T / 2$ (the proof goes along the same lines in the other case).

Let $t_{0}:=T$ if $\forall t \in[0, T), s_{0}(t) \leq \sqrt{\left(2 n+\frac{11}{6}\right) \pi}$. Otherwise, let $t_{0}<T$ be such that: $\forall t \in\left[0, t_{0}\right), s_{0}(t) \leq$ $\sqrt{\left(2 n+\frac{11}{6}\right) \pi}$ and $s_{0}\left(t_{0}\right)=\sqrt{\left(2 n+\frac{11}{6}\right) \pi}$.

By (106) and (117), we have $b_{n+1}-b_{n}<\delta$. The function $\alpha$ has been defined in (119) such that on $[0, T]$, $\alpha(t) \in\left[b_{n}, b_{n+1}\right]$. Moreover, $t_{0}$ has been defined so that on $\left[0, t_{0}\right], s_{0}(t) \in\left[b_{n}, b_{n+1}\right]$. Therefore, we can define a measurable disturbance $d_{1}: \mathbb{R}^{+} \rightarrow \mathbb{R}$ essentially bounded by $\delta$ such that:

$$
\forall t \in\left[0, t_{0}\right], d_{1}(t)=\alpha(t)-s_{0}(t)
$$

The definition of $d_{1}$ and (120) show that there exists some trajectory $X_{1}$ of the closed-loop system

$$
\dot{x}=f\left(x, u\left(x+d_{1}, t\right)\right)
$$

with the initial condition $X_{1}(0)=x_{n}$ such that

$$
\forall t \in\left[0, t_{0}\right], X_{1}(t)=s_{0}(t)
$$

If $t_{0}=T$, Lemma 7 shows that $X_{1}(T)>x_{n}$. Otherwise, $X_{1}\left(t_{0}\right)=\sqrt{\left(2 n+\frac{11}{6}\right) \pi}$, then integrate (123) between $t_{0}$ and $T$, using estimates (112) and (114), as well as (109), to find that

$$
X_{1}(T)>x_{n} .
$$

Now, either $\forall t \geq T, X_{1}(t)>x_{n}$, then take $d=d_{1}$ and $x=X_{1}$, the function $x$ is a trajectory of the closed-loop system

$$
\dot{x}=f(x, u(x+d, t))
$$


and its limsup is greater than $x_{n}$.

Or there is a time $\tau_{1}>T$ such that $X_{1}\left(\tau_{1}\right)=x_{n}$. Let $t_{1}=\tau_{1}+T$ if $\forall t \in\left[\tau_{1}, \tau_{1}+T\right), s_{0}(t) \leq \sqrt{\left(2 n+\frac{11}{6}\right) \pi}$.

Otherwise, let $t_{1}<T$ be such that: $\forall t \in\left[\tau_{1}, \tau_{1}+t_{1}\right), s_{0}(t) \leq \sqrt{\left(2 n+\frac{11}{6}\right) \pi}$ and $s_{0}\left(t_{1}\right)=\sqrt{\left(2 n+\frac{11}{6}\right) \pi}$.

Then let $d_{2}$ be a measurable measurement disturbance essentially bounded by $\delta$ such that

$$
\forall t \in\left[0, \tau_{1}\right), d_{2}(t)=d_{1}(t)
$$

and

$$
\forall t \in\left[\tau_{1}, \tau_{1}+t_{1}\right), d_{2}(t)=\alpha(t)-s_{\tau_{1}}(t) .
$$

The definition (120) of $s_{\tau_{1}}$ shows that there exists some trajectory $X_{2}$ of the closed-loop system

$$
\dot{x}=f\left(x, u\left(x+d_{2}, t\right)\right.
$$

with the initial condition $X_{2}(0)=x_{n}$, and such that

$$
\forall t \in\left[0, \tau_{1}\right), X_{2}(t)=X_{1}(t)
$$

and

Therefore, like above, $X_{2}\left(\tau_{1}+T\right)>x_{n}$.

$$
\forall t \in\left[\tau_{1}, \tau_{1}+t_{1}\right], X_{2}(t)=s_{\tau_{1}}(t) .
$$

By repeating this process as many times as needed, we can define a measurable measurement disturbance $d$ bounded by $\delta$ and some trajectory $x$ of the closed-loop system

$$
\dot{x}=f(x, u(x+d, t))
$$

such that $x(0)=x_{n}$, and

$$
\forall t \geq 0, x(t)=x_{n} \Rightarrow x(t+T)>x_{n} .
$$

This implies that $\varlimsup_{t \rightarrow \infty} x(t) \geq x(0)$.

When $\delta$ is given, this construction can be done for any large enough $n$. Moreover, the construction can be done for any positive $\delta$. We can therefore conclude in the same way as in Section 3 that the system cannot be made ISS.

\section{REFERENCES}

[1] E.D. Sontag, Smooth stabilization implies coprime factorization. IEEE Trans. Automat. Cont. 34 (1989) $435-443$.

[2] R. Freeman, Global internal stabilizability does not imply global external stabilizability for small sensor disturbances. IEEE Trans. Automat. Contr. 40 (1996) 2119-2122.

[3] R. Freeman and P. Kokotovic, Robust nonlinear control design - state-space and Lyapunov techniques, Birkhäuser, Boston Basel Berlin (1996).

[4] E.D. Sontag, Mathematical control theory: Deterministic Finite Dimensional Systems, Text in Applied Mathematics 6, Springer-Verlag, New York Berlin Heidelberg (1990).

[5] C. Samson, Velocity and torque feedback control of a nonholomic cart, in Robot Control, Proc. of the International Workshop on Nonlinear and Adaptive Control: Issues in Robotics, C. Canudas de Wit Ed., Grenoble, France, November 21-23, 1990, Springer-Verlag, Berlin Heidelberg New York, Lecture Notes in Control and Information Sciences 162 (1991) $125-151$.

[6] J.M. Coron, Global asymptotic Stabilization for controllable systems without drift. Math. Control Signals Systems 5 (1992) 295-312.

[7] J.M. Coron, Stabilization in finite time of locally controllable systems by means of continuous time-varying feedback laws. SIAM J. Contr. Opt. 33 (1995) 804-833.

[8] J.M. Coron, On the stabilization of controllable and observable systems by an output feedback law. Math. Control Signals Systems 7 (1994) 187-216.

[9] R. Freeman, Time-varying feedback for the global stabilization of nonlinear systems with measurement disturbances, in Proc. European Control Conference, Brussels (1997). 
[10] N.N. Krasovskii, Stability of motion, Standford University Press, Standford (1963).

[11] J.M. Coron, L. Praly and A. Teel, Feedback stabilization of nonlinear systems: sufficient conditions and Lyapynov and Inputoutput techniques, in Trends in Control, A. Isidori Ed., Springer-Verlag (1995) 293-348.

[12] E.D. Sontag and Y. Wang, New characterizations of the input to state stability property. IEEE Trans. Automat. Contr. 41 (1996) 1283-1294.

[13] Y. Lin, Input-to-state stability for noncompact sets Proc. 13th IFAC World Congress, Vol. E, San Francisco (1996) 73-78. 